\title{
The anti-snoring bed - a pilot study
}

\author{
Elisabeth Wilhelm ${ }^{1 *}$ (D), Francesco Crivelli ${ }^{1,2}$, Nicolas Gerig ${ }^{1,3}$, Malcolm Kohler ${ }^{4}$ and Robert Riener ${ }^{1,5}$
}

\begin{abstract}
Purpose: Avoiding supine position can reduce snoring in most habitual snorers. However, devices that restrict the sleeping position cause discomfort or disrupt sleep resulting in low compliance. Therefore, mechanisms, which lift the trunk of the user without disturbing sleep, have been proposed. We present the first study, which investigates whether individual interventions provided by beds with lifting mechanisms are able to stop snoring (success rate) and whether they reduce the snoring index (number of total snores divided by total time in bed) using a repeated measures design. In addition, we investigated whether the intervention is interfering with the subjective sleep quality.

Methods: Twenty-two subjects were observed for four nights (adaptation, baseline, and two intervention nights). During intervention nights, the bed lifted the trunk of the user in closed-loop manner. Subjects were divided in three groups (non-snorer, snorer one, and snorer two). Non-snorers were lifted by the bed at random time points during the night. In group snorer one, a stepwise increase of the bed inclination was compared with going directly to a randomly selected angle. In group snorer two, the influence of a small inclination angle $\left(10^{\circ}\right)$ and a big inclination angle $\left(20^{\circ}\right)$ was compared.
\end{abstract}

Results: Snoring was stopped successfully in 22\% (small angle) and 67\% (big angle) of the interventions. This did not lead to a significant reduction in the snoring index. The subjective sleep quality was not reduced by the intervention.

Conclusion: The anti-snoring bed is able to stop individual episodes of habitual snoring without reducing the subjective sleep quality.

Trial Registration: https://clinicaltrials.gov, no. NCT04053738, registered 12 August 2019 - Retrospectively registered, https://clinicaltrials.gov/ct2/show/NCT04053738.

Keywords: Habitual snoring, Intelligent bed, Positional therapy

\section{Introduction}

Habitual snoring is a widespread sleep problem (Deary et al. 2014), which does not only affect the health of the snorer (Endeshaw et al. 2013) but also the quality of life of the bed partner (Beninati et al. 1999). Intense snorers snore up to 245 times/hour (Cathcart et al. 2010). Snoring often occurs when the muscle tone drops while the snorer is lying in supine position. In this position, gravitational forces pull the soft tissue surrounding the upper airways in dorsal direction, thereby narrowing the

*Correspondence: elisabeth.wilhelm@hest.ethz.ch

'Sensory-Motor Systems Lab, Department of Health Sciences and Technology,

Eidgenossische Technische Hochschule Zurich, Zurich, Switzerland

Full list of author information is available at the end of the article airways. This causes audible flow turbulences in the upper airways (Gleadhill et al. 1991).

Therapeutic interventions force snorers to avoid supine position. The easiest way to implement positional therapy is to attach an obstacle, to the back of the snorer (Berger et al. 1997). More sophisticated devices include vibration (van Maanen and de Vries 2014) or sound (Cartwright et al. 1985) alarms raised when the snorer sleeps in supine position, or cushions, which push the head of the snorer sideward (Zhang et al. 2013). However, the discomfort needed to force the snorer to avoid supine position results in disrupted sleep and low user compliance (Bignold et al. 2009). 
An alternative approach is to elevate the trunk of the user with cushions. McEvoy et al. reported that sleeping in seated position (trunk elevation of $60^{\circ}$ ) has positive influences on the severity of obstructive sleep apnea (McEvoy et al. 1986). Recently it has been suggested to use adjustable bed frames to change the position of the sleeping snorer (Van Der Loos et al. 2003). Actuated beds such as the one used within this paper cost about 7000 Swiss francs and are therefore more expensive than conventional approaches for providing positional therapy. Commercially available versions such as the Partner Snore TM Technology (Sleep Number Corporation, Minneapolis, USA) elevate the trunk of the sleeping snorer if the bed partner indicates the presence of snoring via a push button (Sleep Number Corporation). Despite the fact that these beds are commercially available, little is known about the effect of such devices.

In this paper, we present the first systematic pilot study, which investigates the influence of automated trunk elevations on snorers. In absence of any evidence on the effect of different intervention parameters, we decided to conduct a pilot study, which investigates multiple options with three small groups. This pilot study aims on identifying the range of promising intervention options for future investigations. In particular, we were interested, which elevation angles would most likely reduce habitual snoring. Furthermore, we investigated how the bed influences the subjective sleep quality of the user.

\section{Materials and methods}

\section{Setup}

The mattress shape was adapted using a custom made intelligent anti-snoring bed (Fig. 1), which is able to detect snoring sound and change the position of the user whenever snoring occurs (Crivelli et al. 2017; Wilhelm et al. 2017). The bed is triggered depending on the snoring index (SI) defined as number of snores detected within a given time frame. This time frame was set to one minute. The threshold that triggered bed motion was ten snores per minute for the first part of the study. After the interim analysis, this threshold was lowered to four snores per minute.

\section{Subjects}

Twenty-two participants (5 female, age 29.7 years SD: 16.0) participated in the study (Table 1). Participants were not medication free. Alcohol and caffeine consumption was not restricted. Exclusion criteria were pregnancy, previously diagnosed sleep-related breathing disorders, chronic lower back pain, heart insufficiency that might impede sleeping in supine position, and inability to follow the procedures of the study. All subjects received a monetary compensation (CHF 25 per night) to cover travel costs. Daytime sleepiness was recorded using the Epworth Sleepiness Scale (ESS). Ten participants reached an ESS-score above 10, which indicates excessive daytime sleepiness (Johns 1991). Apnea hypopnea index (AHI) and oxygen desaturation index (ODI) were measured using a home-monitoring device (Apnea Link, purchased from ResMed). The scoring was done automatically by the ApneaLink software (purchased from ResMed). Two participants had an AHI and an ODI bigger than 5 during the adaptation night. One of these two participants also scored more than 10 points in the ESS. Therefore, we cannot exclude that these participants suffer from an undiagnosed sleep-related breathing disorder. In addition,
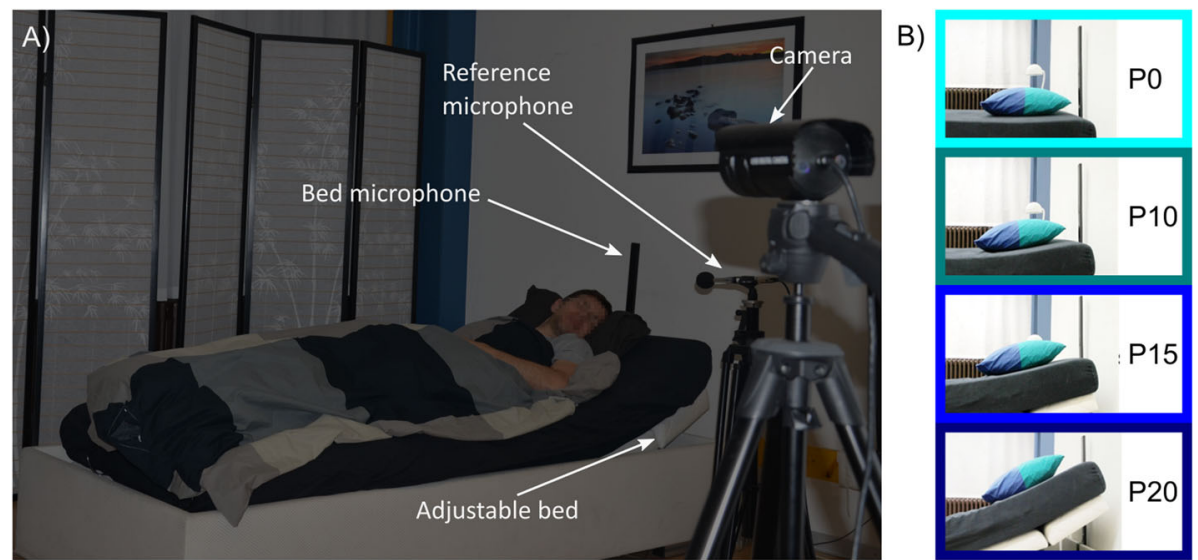

Fig. 1 Measurement setup. a Postural interventions were provided using an automated bed, which can elevate the trunk of the sleeping user. A custom-made built-in microphone was used to trigger trunk elevation. In addition, a reference microphone and a IR-camera were used to monitor the user. $\mathbf{b}$ The inclination angles provided by the bed were $10^{\circ}(\mathrm{P} 10), 15^{\circ}(\mathrm{P} 15)$, and $20^{\circ}(\mathrm{P} 20)$ provided at the location where the users hip joint is to be expected. At the beginning of the night on during episodes of silence the bed was set to neutral postion (P0) 
Table 1 Demographics of the study groups. Supine Sleeping was calculated as the percentage of the total time in bed that was spent in supine position. Position dependency was assumed if more than $50 \%$ of all snores during the baseline night occurred in supine position. AHI and ODI have been obtained using the automatic scoring provided by the ApneaLink software (purchased from ResMed). Age, BMI, ESS, AHI, and ODI are reported as mean and standard deviation. All other values indicate the number of subjects

\begin{tabular}{|c|c|c|c|c|c|c|}
\hline & \multicolumn{2}{|c|}{ Non-snorer } & \multicolumn{2}{|l|}{ Snorer 1} & \multicolumn{2}{|l|}{ Snorer 2} \\
\hline & recruited & datasets & recruited & datasets & recruited & datasets \\
\hline Subjects & 7 & 6 & 6 & 4 & 9 & 6 \\
\hline $\operatorname{Sex}(m / f)$ & $5 / 2$ & $4 / 2$ & $5 / 1$ & $4 / 0$ & $7 / 2$ & $5 / 1$ \\
\hline Age (years) & $24.9(4.1)$ & $24.8(4.5)$ & $25.8(4.0)$ & $26.0(5.1)$ & $37.8(21.9)$ & $30.3(15.6)$ \\
\hline BMI $\left(\mathrm{kg} / \mathrm{m}^{2}\right)$ & $22.6(2.5)$ & $22.8(2.6)$ & $21.9(1.7)$ & $21.1(1.3)$ & $24.8(4.3)$ & $25.2(5.2)$ \\
\hline ESS (score 0-24) & $10.9(3.5)$ & $9.9(2.5)$ & $8.2(3.4)$ & $6.8(3.3)$ & $9.3(4.0)$ & $9.7(3.1)$ \\
\hline Supine Sleeping (\%) & - & $52(20)$ & - & $48(11)$ & - & $34(15)$ \\
\hline Coffee (Cups per day) & - & $2.0(2.0)$ & - & $1.0(0.7)$ & - & $1.1(1.2)$ \\
\hline Alcohol (Glasses per day) & - & $0.6(0.6)$ & - & $0.25(0.3)$ & - & $0.5(0.5)$ \\
\hline AHI adaptation (n/hour) & - & $0.95(0.75)$ & - & $3.28(5.75)$ & - & $2.83(3.26)$ \\
\hline ODI adaptation (n/hour) & - & $1.63(1.12)$ & - & $2.78(4.68)$ & - & $3.67(4.37)$ \\
\hline Smokers & 0 & 0 & 0 & 0 & 3 & 2 \\
\hline Position dependency & n.a. & n.a. & 4 & 3 & 6 & 3 \\
\hline
\end{tabular}

participants were asked to report any breathing related comorbidity. One participant reported being allergic to cats and having frequent breathing problems after smoking for about 6 years. Another participant reported being affected by allergic rhinitis (hay fever). Four participants reported experiencing symptoms of a cold on one or more days on which they participated in the study.

Participants were divided into snorers and non-snorers based on the amount of snoring recorded with a homemonitoring device (Apnea Link, purchased from ResMed) during the first night. The threshold was set to 50 snores per night. This value was chosen with respect to the normal breath rate of a human subject (12 -20 breath/minute), someone who snores 50 times per night snores between 2.5 and $5 \mathrm{~min}$ in total. Interventions by the bed are only triggered after 1 minute of continuous snoring. Therefore, the chance to see interventions in snorers who snore less than 50 times per night would be very low. Furthermore, by excluding subjects with a snoring episode of less than 5 min we wanted to avoid overestimation of an overestimation of positive interventions. Positive interventions were defined as bad movements (which are triggered after 1 minute of snoring) that were able to stop snoring within 3 minutes time. Participants were recruited in two phases. The group snorer one was recruited between February and July 2017. The group snorer two was recruited from February to July 2018. All non-snorers recruited in both recruitment periods received the same interventions. Parameters for snorers were optimized after completion of the recruitment period.
One subject withdrew from the study. Four subjects did not receive the intervention in at least one night of the study due to technical problems. Three snorers did not trigger the bed. These datasets were excluded from the evaluation. Some subjects only triggered the bed in one of the experimental conditions. Nights in which the bed was not triggered were treated as missing data points.

\section{Study protocol}

All subjects spent four consecutive nights in the lab. Daytime activities were not restricted. Subjects went to bed at their habitual bedtime. The first night served as adaptation night. Night two to four were used as experimental nights. These nights were split into one baseline night and two nights with interventions. The order of the nights was randomized.

Within this study, we used four different elevation angles $(0,10,15$, and 20 degree referred to as P0, P10, P15, $\mathrm{P} 20$, respectively). The bed moved with an angular velocity of $1.5 \%$ s. As depicted in Fig. 2 the three groups got different interventions.

Within the groups snorer one and non-snorer we wanted to investigate whether a stepwise increasing inclination angle (stepwise condition) is less disturbing than a sudden change of position (random angle condition) (Fig. 2). Since Non-snorers would not trigger the bed with snoring sounds, interventions were provided to them at random time points throughout the night.

We compared the lowest angle $\left(10^{\circ}, \mathrm{P} 10\right)$ with the highest inclination angle $\left(20^{\circ}, \mathrm{P} 20\right)$ used within the group snorer two. Nights during which the bed only went to P10 


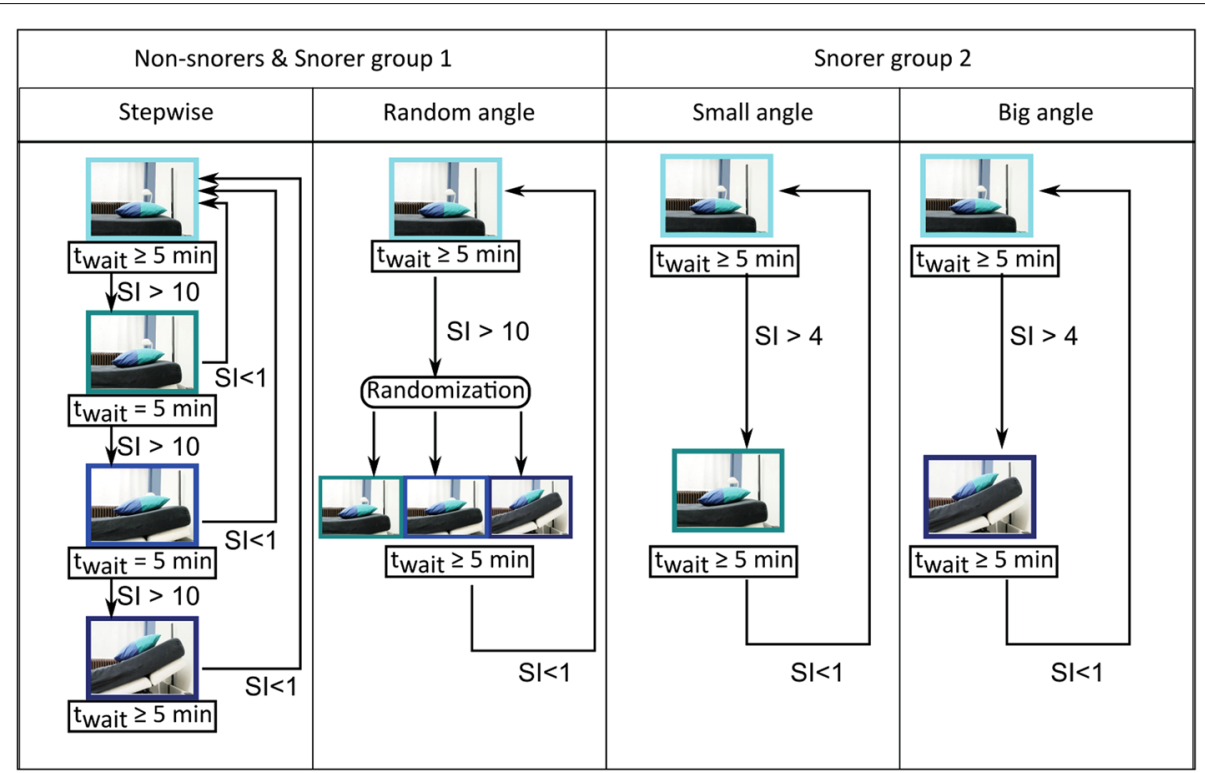

Fig. 2 Interventions provided by the bed. Participants of the groups snorer one and non-snorer were provided with a stepwise intervention and a random angle intervention. During stepwise intervention, the bed was elevated upon the detection of snoring. If snoring stopped within 5 minutes, the bed went back to P0. Otherwise, the inclination was increased to the next step. During the random angle intervention, the bed went to one of the three inclination angles upon detection of snoring and waited in this position until snoring stopped. The group snorer two got two different interventions. During the small angle intervention, the bed moved to P10 upon detection of snoring and back to neutral when snoring stopped. During the big angle intervention, the bed went to P20

are called small angle. The other nights are referred to as big angle.

\section{Goal of the study}

Within this study, we investigated, whether individual interventions of the bed are able to stop snoring episodes. In addition, we were interested, whether the overall amount of snoring would be reduced in intervention nights compared to baseline. Furthermore, we investigated whether the bed influences the subjective sleep quality.

Snoring activity was monitored using a portable homemonitoring device (Apnea Link plus, purchased from ResMed, Switzerland). Since audio based detection has been reported to be the most reliable way of snoring detection (Arnardottir et al. 2016), we also used a sound level meter (XL2 Audio and acoustic Analyzer, purchased from NTi Audio, Lichtenstein). Manual visual-audio scoring of the data collected with the noise level meter was performed using the XL2 data explorer.

The success rate of the individual intervention was derived by dividing the number of positive interventions through the total number of bed movements.

To investigate the effect on the total amount of snoring observed within one night, we calculated the SI using the lables of the software of the Apnea Link and the lables of the manual scoring. The SI was calculated by dividing the number of snores $(S)$ through the recording time $\left(t_{\text {record }}\right)$ :

$$
S I=\frac{\sum_{i} S}{t_{\text {record }}}
$$

The sleeping position of the user was manually scored based on an infrared video. We distinguished between supine, prone, left, or right position and movement episodes.

Subjective Sleep Quality was assessed using the Groningen Sleep Quality Scale (GSQS) (Meijman et al. 1988). In addition, subjects were asked to indicate whether they think that an intervention occurred during the night, whether they woke up due to an intervention, and whether the intervention was disturbing their sleep. On the morning after the last experimental night, subjects were also asked to indicate which condition they preferred. The questionnaires were filled out within the first 10 minutes after wake up.

\section{Statistical analysis}

The data was analysed using Matlab r2018b (MathWorks, USA). Data was tested for normality using the Kolmogorow-Smirnow-Test. Since this test indicated 
a non-normal distribution, we used a non-parametric method. The Friedman-Test, which would be the nonparametric alternative for an ANOVA in repeated measures design, could not be used, since it does not accept missing values. Alternative tests, which do accept missing values, require a bigger sample size. Therefore, we used the Kruskal-Wallis Test to compare the values within each group. All results are reported as mean and range. The significance level was set to $p=0.05$.

We decided to look at each group individually during the analysis, because the number of bed movements differed in between groups.

\section{Results}

\section{Number of interventions triggered by different groups}

Non-snorers received 12 bed movements in both random angle and stepwise condition. The group Snorer one triggered the bed on average 1.5 (range: 0,4 ) times and 2 (range: 0,7 ) times in stepwise and random angle condition, respectively. In stepwise condition, all but one snorer stopped snoring when the bed reached P10. The other snorer stopped snoring when the bed was in P15. Averaged over all nights snorers of the group snorer one only encountered 6.3 (range: 0,13 ) bed movements. This is only half of what the control group experienced. Therefore, we decided to investigate at each group individually.

Participants of the group snorer two triggered the bed on average 3 (range: 0,11 ) and 2 (range: 0,6 ) times in the small and big angle condition, respectively.

\section{Effects of the postural intervention on snoring}

In the group snorer one $60.0 \%$ of the stepwise interventions and $57.0 \%$ of the random angle interventions were successful. As depicted in Fig. 3 the bed reduced the audio-based SI slightly in snorer one during the random angle condition (n.s.). In the data recorded with the homemonitoring device there was no reduction of snoring in intervention nights.

In the group snorer two, small angle was provided 18 times with a success rate of $22.2 \%$. The intervention big angle was triggered 12 times with a success rate of $66.6 \%$. There was no statistical significant difference between the audio-based SI recorded during the different nights. The same applied for the SI measured with the home-monitoring device. However, both outcome measures showed a tendency towards a lower SI in the big angle condition (see Fig. 3).

\section{Effects of the postural intervention on subjective sleep quality}

As depicted in Fig. 4 the stepwise intervention seemed to decrease subjective sleep quality measured using the GSQS in the groups non-snorer and in snorer one (n.s.). Participants of both groups noticed in which nights interventions occurred. This result was significant in snorer one ( $p=0.0498, \tilde{\chi}^{2}=6.0000$, with a mean rank of 7.5, 3.0, and 3.0 for baseline, stepwise, and random angle respectively) but not in non-snorer. The questions whether subjects woke up due to an intervention and whether the bed did disturb the participants did not give significant results. Two out of four snorers and two out of six non-snorers did not indicate which condition they preferred. One out of six non-snorer chose the stepwise condition and one the random angle condition. All other participants indicated that they preferred the baseline condition.

In the group snorer two, we investigated the influence of small and big inclination angles. As depicted in Fig. 4. there was no difference in GSQS. Subjects of snorer two noticed in which nights interventions occurred ( $p=$ $0.0481, \tilde{\chi}^{2}=6.0701$ ) with a mean rank of $13.67,8.17$, and 6.67 for baseline, small angle, and big angle, respectively. Furthermore, they reported that they had been awake due to the intervention $\left(p=0.0439, \tilde{\chi}^{2}=6.2503\right.$, mean rank of 5.3, 10.25, and 12.92 for baseline, small angle, and big angle, respectively). However, the question whether the intervention disturbed their sleep was not giving significant results. Three out of six participants of the group snorer two reported that the big angle condition was their favourite condition, two opted for baseline, and one for small angle condition.

\section{Discussion}

Within our study, snoring stopped in $60 \%, 57 \%$, or $67 \%$, while a intervention in stepwise, random angle, or big angle, was provided, respectively. However, this did not lead to a significant reduction of the average SI. Eventually, the natural fluctuation of snoring covered the effect of the individual interventions. Within the same subject snoring frequency and the duration of snoring episodes can vary by $22 \%$ and $33 \%$, respectively, in consecutive nights (Cathcart et al. 2010). Therefore, long-term studies might be needed to evaluate the effect of anti-snoring beds. While the natural variation of habitual snoring makes it hard to evaluate scientific studies, it also shows how important it is to provide interventions in closed-loop manner. The closed-loop approach allows the user to sleep in the sleeping position he prefers and will only intervene if snoring occurs. Therefore, it is much less restrictive than commonly used approaches.

In addition, our study had some limitations. In some nights, the nasal cannula was displaced due to movement. In these cases, the SI was only calculated for periods with valid measurements. Since the snoring activity varies throughout the different sleep stages this reduces the validity of this measure. The audio-based evaluation has been reported to be more reliable. However, due to the absence of a clear definition of snoring sound, this 

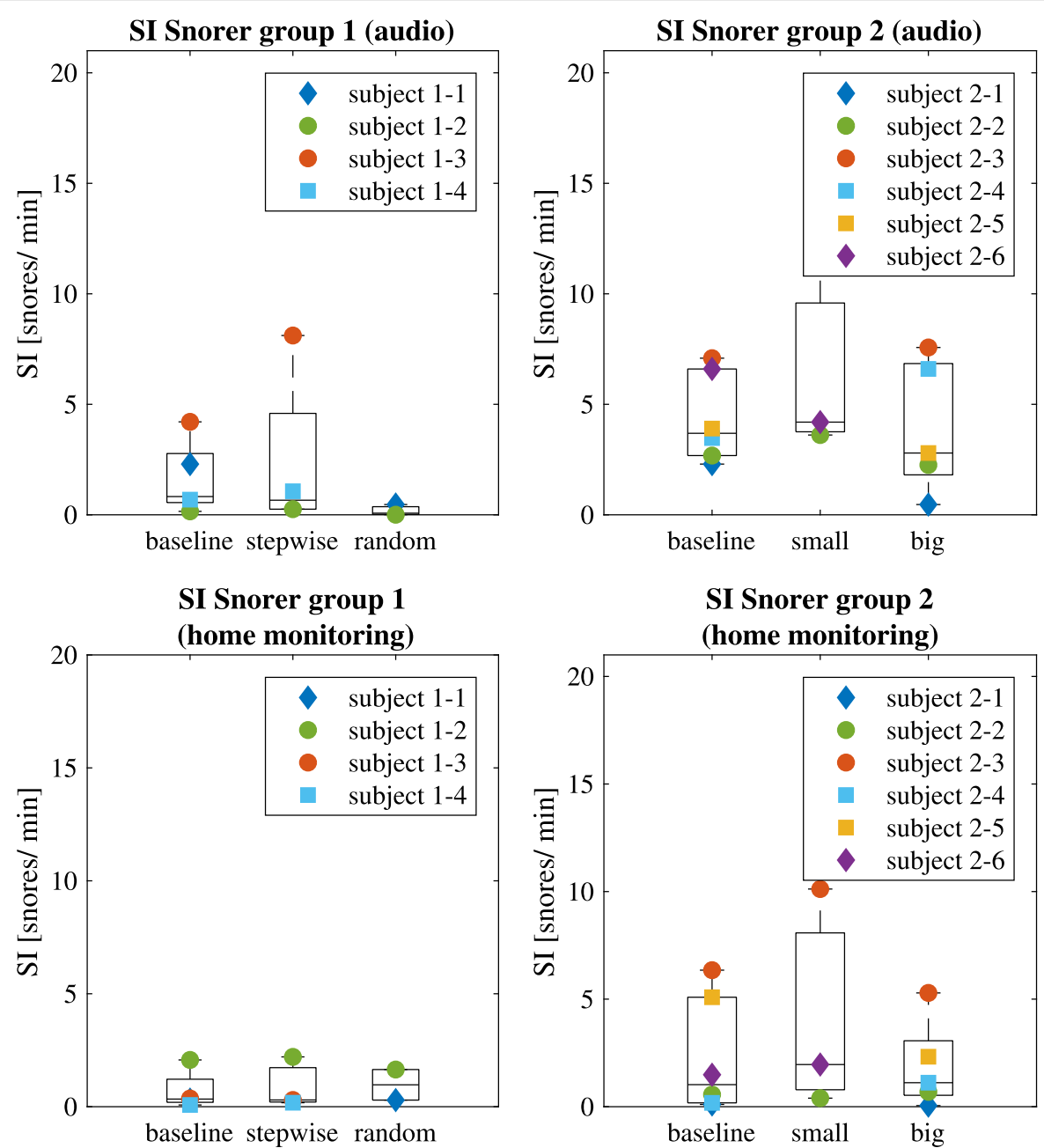

Interventions stopping snoring
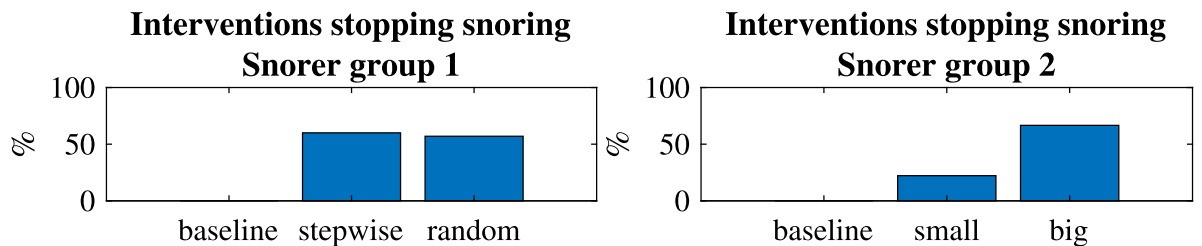

Fig. 3 Effect of the automated bed on snoring. The average SI was once derived from manually scored audio-recording and once from the nasal cannula of the home-monitoring device. As depicted the bed was able to stop snoring with a success rate of $22.2 \%$ to $66.6 \%$ in different test conditions. However, the successful interventions did not lead to a reduction of the average SI which describes the total amount of snores divided by the time spent in bed

evaluation is influenced by the subjective experience of the expert scorer (Rohrmeier et al. 2014).

Furthermore, there are multiple causes for habitual snoring. We recruited snorers based on self-report. Therefore, we had a mixed population. So far, it has been suggested that anti-snoring beds can only be effective for position dependent snorers. One reason why we did not see significant changes of the SI could be that both our snorer groups did also contain snorers who were not position dependent.
Another limitation of our study is, that in non-snorers interventions were provided regardless of the sleeping position. Position dependent snorers would be expected to trigger the bed while lying in supine position. In the non-snorers in our study only $24 \%$ of the upward movements of the stepwise condition and $47 \%$ of the upward movements of the random angle condition occurred while the participants were lying in supine position. This might be due to the fact that only three out of the six participants of this groups spent more than $50 \%$ of the baseline 


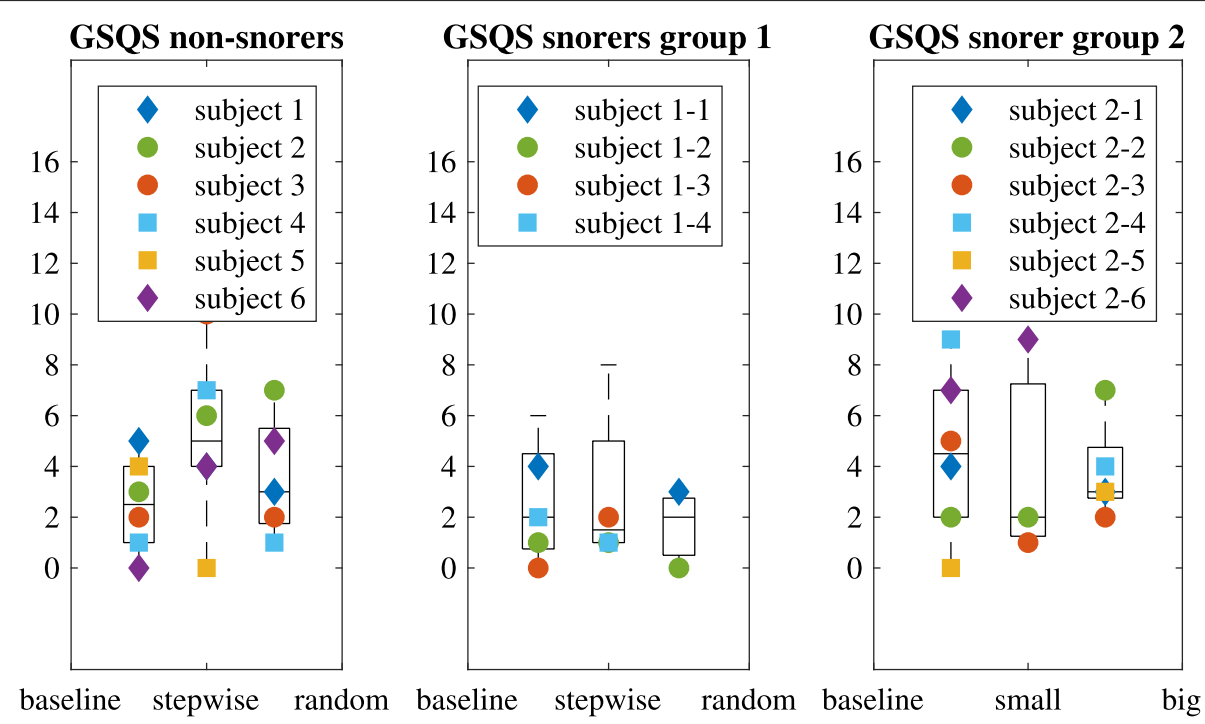

Fig. 4 Influence on subjective sleep quality. GSQS scores reach from 0 to 14 with 0 indicating good sleep and 14 indicating bad sleep. As depicted non-snorers slept worse during intervention nights. In group snorer one sleep quality improved slightly during the random angle condition. The group snorer two had a tendency to report better sleep quality in both intervention conditions. None of these results was significant

night in supine position. By recruiting non-snorers who are mainly sleeping in supine position, one could investigate the effect of the intervention in this specific sleeping position.

In addition, the snoring detection algorithm of the antisnoring bed did not recognize all types of snoring. Therefore, some snorers did not trigger the bed. Eight times the bed reacted to environmental noise. This implies that more robust snoring detection algorithms could decrease sleep disturbance caused by anti-snoring beds. Alternatively, the interventions provided by the bed could be triggered manually by an expert scorer. However, this solution would be very cost intensive and could therefore be only used in scientific studies and not as a long-term treatment for habitual snoring.

With respect to the effect of anti-snoring beds on subjective sleep quality, we saw a slight tendency towards an increased subjective sleep quality in snorers in conditions in which the bed moved to a target position and back. In the stepwise condition, the subjective sleep quality decreased. This might be because the bed moved more frequently in this condition. The effect of an intervention on the objective sleep quality can only be investigated using polysomnography. However, polysomnography is highly obtrusive and would reduce the subjective sleep quality of the participants. As reduced comfort is the most common reason for aborting conventional therapy approaches, our approach would only be a real alternative if the subjective sleep quality is at least similar to baseline condition. Therefore, we decided not to investigate objective sleep quality measures and did instead focus on the subjective sleep quality in our pilot study. In future studies polysomnography should be included to investigate whether the motion of the bed causes arousals or changes the sleep architecture.

\section{Conclusion}

In our Anti-snoring bed study, snoring stopped more frequently when interventions of the type big angle $\left(20^{\circ}\right)$ were provided compared to interventions of the type small angle $\left(10^{\circ}\right)$. This suggests that larger inclination angles might be more efficient. Furthermore, conditions with less frequent bed movements that cover a big range of motion seem to have less effect on subjective sleep quality than conditions with multiple smaller bed movements. The interventions provided by the bed did not lead to a significant reduction in subjective sleep. Further studies are needed to investigate whether Anti-snoring beds are a valuable alternative to conventional positional therapy.

\section{Abbreviations}

BMI: Body Mass Index; CHF: Swiss Franc; ESS: Epworth Sleepiness Scale; f: Female; IR: Infra red; m: Male; n.s.: not significant; P0: Elevation angle $0{ }^{\circ}$; P10: Elevation angle $10^{\circ}$; P15: Elevation angle $15^{\circ}$; P20: Elevation angle $20^{\circ}$; S: Number of snores; SD: Standard deviation; SI: Snoring index; $t_{\text {record: }}$ Recording time; $t_{\text {wait }}$ : Wait time; GSQS: Groningen Sleep Quality Scale; AHI: Apnea Hypopnea Index; ODI: Oxygen Desaturation Index

\section{Acknowledgements}

We thank François Pugliese from Elite SA for his support throughout the project. In addition, we thank Michael Herold-Nadig and Marco Bader from ETH Zurich for their technical support, their advice and contribution during the design and the development of the devices. Furthermore, we thank Rachel van Sluijs, Antonino Crivello, Quincy Rondei, and Alexander Breuss for their support during the measurements. We also thank Jana Petr for her support during sound file scoring. 


\section{Authors' contributions}

The study was designed by FC, RR, and MK. Device and sensor setup were developed by FC, EW, NG, and RR. Data processing and analysis was done by $N G, M K$ and EW. The manuscript was written by EW. The manuscript was critically reviewed by all co-authors. The author(s) read and approved the final manuscript.

\section{Funding}

This work was supported by the Commission for Technology and Innovation (CTI) under grant No. 17988.1 PFIW-IW, the company Elite SA, and by the Swiss National Science Foundation (SNF) under grant No. 162809.

\section{Availability of data and materials}

Video and audio data generated during the current study are not publicly available, because this data could not be anonymized. Tables containing the GSQS, the snoring indices, the manual scoring of the audio data of the snorers, the manual scoring of the video data of the snorers, and the scores obtained with the home-monitoring device are available on reasonable request in the ETH research collection repository under https://www.research-collection. ethz.ch/handle/20.500.11850/396977.

\section{Ethics approval and consent to participate}

The experimental procedure was approved by the Ethics Committee of ETH Zurich, no. EK 2015-N-70. Written informed consent was obtained from all subjects.

\section{Consent for publication}

The person depicted in figure one signed consent stating that the picture can be used in this publication.

\section{Competing interests}

This study was conducted within an industry collaboration project with Elite SA. Elite SA contributed to the design of the device and the study, but not to data collection and analysis, decision to publish, or preparation of the manuscript.

\section{Author details}

${ }^{1}$ Sensory-Motor Systems Lab, Department of Health Sciences and Technology, Eidgenossische Technische Hochschule Zurich, Zurich, Switzerland. ${ }^{2}$ CSM SA, Center Alpnach, Robotics \& Automation, Alpnach Dorf, Switzerland. ${ }^{3}$ Bio-Inspired RObots for MEDicine-Lab (BIROMED-Lab), Department of Biomedical Engineering, University of Basel, Basel, Switzerland. ${ }^{4}$ Department of Pulmonology, University Hospital Zurich, Zurich, Switzerland. ${ }^{5}$ Spinal Cord Injury Center, University Hospital Balgrist, Zurich, Switzerland.

Received: 17 February 2020 Accepted: 13 August 2020

Published online: 08 September 2020

\section{References}

Arnardottir ES, Isleifsson B, Agustsson JS, Sigurdsson GA, Siqurgunnarsdottir MO, Sigurdarson GT, Saevarsson G, Sveinbjarnarson AT, Hoskuldsson S, Gislason T. How to measure snoring? a comparison of the microphone, cannula and piezoelectric sensor. J Sleep Res. 2016;25(2):158-68.

Beninati W, Harris CD, Herold DL, Shepard Jr JW. The Effect of Snoring and Obstructive Sleep Apnea on the Sleep Quality of Bed Partners. Mayo Clinic Proceedings. 1999;74(10):955-8. https://doi.org/10.4065/74.10.955. http:// www.sciencedirect.com/science/article/pii/S0025619611639918.

Berger M, Oksenberg A, Silverberg D, Arons E, Radwan H, laina A. Avoiding the supine position during sleep lowers $24 \mathrm{~h}$ blood pressure in obstructive sleep apnea (osa) patients. J Hum Genet. 1997;11(10):657-64.

Bignold JJ, Deans-Costi G, Goldsworthy MR, Robertson CA, McEvoy D, Catcheside PG, Mercer JD. Poor long-term patient compliance with the tennis ball technique for treating positional obstructive sleep apnea. J Clin Sleep Med. 2009;5(05):428-30.

Cartwright RD, Lloyd S, Lilie J, Kravitz H. Sleep position training as treatment for sleep apnea syndrome: a preliminary study. Sleep. 1985;8(2):87-94.

Cathcart R, Hamilton D, Drinnan M, Gibson G, Wilson J. Night-to-night variation in snoring sound severity: one night studies are not reliable. Clin Otolaryngol. 2010;35(3):198-203.

Crivelli F, Wilhelm E, van Sluijs R, Riener R. Actuated bed for a closed loop anti-snoring therapy. In: 2017 International Conference on Rehabilitation Robotics (ICORR). London: IEEE; 2017. p. 823-7.
Deary V, Ellis JG, Wilson JA, Coulter C, Barclay NL. Simple snoring: not quite so simple after all? Sleep Med Rev. 2014;18(6):453-62.

Endeshaw Y, Rice TB, Schwartz AV, Stone KL, Manini TM, Satterfield S, Cummings S, Harris T, Pahor M, Study HA. Snoring, daytime sleepiness, and incident cardiovascular disease in the health, aging, and body composition study. Sleep. 2013;36(11):1737-45.

Gleadhill IC, Schwartz AR, Schubert N, Wise RA, Permutt S, Smith PL. Upper airway collapsibility in snorers and in patients with obstructive hypopnea and apnea. Am Rev Respir Dis. 1991;143(6):1300-3.

Johns MW. A new method for measuring daytime sleepiness: the epworth sleepiness scale. Sleep. 1991;14(6):540-5.

McEvoy RD, Sharp DJ, Thornton AT. The effects of posture on obstructive sleep apnea. Am Rev Respir Dis. 1986;133(4):662-6.

Meijman T, de Vries-Griever A, De Vries G, Kampman R. The evaluation of the groningen sleep quality scale. Groningen: Heymans Bull (HB 88-13-EX). 1988;2006:

Rohrmeier C, Herzog M, Ettl T, Kuehnel TS. Distinguishing snoring sounds from breath sounds: a straightforward matter? Sleep Breathing. 2014;18(1): 169-76.

Sleep Number Corporation. FlexFit" smart adjustable bases. https://www. sleepnumber.com/sn/en/c/bed-bases. Accessed 17 Feb 2019.

Van Der Loos HM, Ullrich N, Kobayashi H. Development of sensate and robotic bed technologies for vital signs monitoring and sleep quality improvement. Auton Robot. 2003;15(1):67-79.

van Maanen JP, de Vries N. Long-term effectiveness and compliance of positional therapy with the sleep position trainer in the treatment of positional obstructive sleep apnea syndrome. Sleep. 2014;37(7):1209-15.

Wilhelm E, Crivelli F, Markic S, Kohler M, Pugliese F, Riener R. Ein intelligentes bett zur verminderung von schnarchen. Somnologie. 2017:21(S2):52.

Zhang J, Zhang Q, Wang Y, Qiu C. A real-time auto-adjustable smart pillow system for sleep apnea detection and treatment. In: 2013 ACM/IEEE International Conference on Information Processing in Sensor Networks (IPSN). Philadelphia: IEEE; 2013. p. 179-90.

\section{Publisher's Note}

Springer Nature remains neutral with regard to jurisdictional claims in published maps and institutional affiliations.

Ready to submit your research? Choose BMC and benefit from

- fast, convenient online submission

- thorough peer review by experienced researchers in your field

- rapid publication on acceptance

- support for research data, including large and complex data types

- gold Open Access which fosters wider collaboration and increased citations

- maximum visibility for your research: over $100 \mathrm{M}$ website views per year

At $\mathrm{BMC}$, research is always in progress.

Learn more biomedcentral.com/submissions 